\title{
First Hitting Problems for Markov Chains That Converge to a Geometric Brownian Motion
}

\author{
Mario Lefebvre ${ }^{\mathbf{1}}$ and Moussa Kounta ${ }^{2}$ \\ ${ }^{1}$ Département de Mathématiques et de Génie Industriel, École Polytechnique de Montréal, C.P. 6079, \\ Succursale Centre-Ville, Montréal, QC, Canada H3C 3 A7 \\ 2 Département de Mathématiques et de Statistique, Université de Montréal, C.P. 6128, \\ Succursale Centre-Ville, Montréal, QC, Canada H3C 3J7
}

Correspondence should be addressed to Mario Lefebvre, mlefebvre@polymtl.ca

Received 1 July 2011; Accepted 21 July 2011

Academic Editors: C.-K. Lin and B. Zhou

Copyright (c) 2011 M. Lefebvre and M. Kounta. This is an open access article distributed under the Creative Commons Attribution License, which permits unrestricted use, distribution, and reproduction in any medium, provided the original work is properly cited.

\begin{abstract}
We consider a discrete-time Markov chain with state space $\{1,1+\Delta x, \ldots, 1+k \Delta x=N\}$. We compute explicitly the probability $p_{j}$ that the chain, starting from $1+j \Delta x$, will hit $N$ before 1 , as well as the expected number $d_{j}$ of transitions needed to end the game. In the limit when $\Delta x$ and the time $\Delta t$ between the transitions decrease to zero appropriately, the Markov chain tends to a geometric Brownian motion. We show that $p_{j}$ and $d_{j} \Delta t$ tend to the corresponding quantities for the geometric Brownian motion.
\end{abstract}

\section{Introduction}

Let $\{X(t), t \geq 0\}$ be a one-dimensional geometric Brownian motion defined by the stochastic differential equation

$$
d X(t)=\mu X(t) d t+\sigma X(t) d B(t)
$$

where $\mu \in \mathbb{R}, \sigma>0$, and $\{B(t), t \geq 0\}$ is a standard Brownian motion. Assume that $X(0)=x \in$ $(1, N)$, where $N \in \mathbb{N}$ (for simplicity), and define

$$
\tau(x)=\inf \{t>0: X(t)=1 \text { or } N \mid X(0)=x\} .
$$

As is well known (see, e.g., Lefebvre [1, page 220]), the probability

$$
p(x):=P[X[\tau(x)]=N]
$$


satisfies the ordinary differential equation

$$
\frac{1}{2} \sigma^{2} x^{2} p^{\prime \prime}(x)+\mu x p^{\prime}(x)=0,
$$

subject to the boundary conditions

$$
p(1)=0, \quad p(N)=1
$$

We easily find that, if $c:=\mu / \sigma^{2} \neq 1 / 2$,

$$
p(x)=\frac{x^{1-2 c}-1}{N^{1-2 c}-1} \quad \text { for } 1 \leq x \leq N
$$

When $c=1 / 2$, the solution is

$$
p(x)=\frac{\ln x}{\ln N} \quad \text { for } 1 \leq x \leq N .
$$

Moreover, the function

$$
m(x):=E[\tau(x)]
$$

satisfies the ordinary differential equation (see, again, Lefebvre [1, page 220])

$$
\frac{1}{2} \sigma^{2} x^{2} m^{\prime \prime}(x)+\mu x m^{\prime}(x)=-1,
$$

subject to

$$
m(1)=m(N)=0 .
$$

This time, if $c \neq 1 / 2$ we find that

$$
m(x)=\frac{2}{(1-2 c) \sigma^{2}}\left\{\ln x-\ln N \frac{x^{1-2 c}-1}{N^{1-2 c}-1}\right\} \quad \text { for } 1 \leq x \leq N
$$

and, for $c=1 / 2$,

$$
m(x)=\frac{\ln x}{\sigma^{2}}(\ln N-\ln x) \quad \text { for } 1 \leq x \leq N .
$$


Now, it can be shown (see Cox and Miller [2, page 213]) that the discrete-time Markov chain $\left\{X_{m \Delta t}, m=0,1, \ldots\right\}$ with state space $\{1,1+\Delta x, \ldots, 1+k \Delta x\}$, where $k$ is such that $1+k \Delta x=N$, and transition probabilities

$$
\begin{gathered}
p_{1+j \Delta x, 1+(j+1) \Delta x}=\frac{1}{2 A}\left\{(1+j \Delta x)^{2} \sigma^{2}+(1+j \Delta x) \mu \Delta x\right\} \\
p_{1+j \Delta x, 1+(j-1) \Delta x}=\frac{1}{2 A}\left\{(1+j \Delta x)^{2} \sigma^{2}-(1+j \Delta x) \mu \Delta x\right\} \\
p_{1+j \Delta x, 1+j \Delta x}=1-\frac{1}{A}(1+j \Delta x)^{2} \sigma^{2}
\end{gathered}
$$

where $j \in\{1, \ldots, k-1\}$, converges to the geometric Brownian motion $\{X(t), t \geq 0\}$ as $\Delta x$ and $\Delta t$ decrease to zero, provided that

$$
\begin{gathered}
(\Delta x)^{2}=A \Delta t, \\
(j \Delta x)^{2}<A \quad \forall j \in\{0, \ldots, k\} .
\end{gathered}
$$

Remarks 1.1. (i) We assume that all the probabilities defined by (1.13) are well defined; that is, they all belong to the interval $[0,1]$.

(ii) The condition in (1.15) implies that $(\Delta x)^{2}<A / k^{2}$.

Let

$$
\begin{gathered}
T_{j}:=\inf \left\{m>0: X_{m \Delta t}=1 \text { or } N \mid X_{0}=1+j \Delta x\right\} \\
p_{j}:=P\left[X_{T_{j}}=N\right] .
\end{gathered}
$$

In the next section, we will compute the quantity $p_{j}$ for $j \in\{1, \ldots, k-1\}$. We will show that $p_{j}$ converges to the function $p(x)$ for the geometric Brownian motion as $\Delta x$ decreases to zero and $k$ tends to infinity in such a way that $1+k \Delta x$ remains equal to $N$. namely,

In Section 3, we will compute the mean number of transitions needed to end the game,

$$
d_{j}:=E\left[T_{j}\right]
$$

By making a change of variable to transform the diffusion process $\{X(t), t \geq 0\}$ into a geometric Brownian motion with infinitesimal mean equal to zero and by considering the corresponding discrete-time Markov chain, we will obtain an explicit and exact expression for $d_{j}$ that, when multiplied by $\Delta t$, tends to $m(x)$ if the time $\Delta t$ between the transitions is chosen suitably.

The motivation for our work is the following. Lefebvre [3] computed the probability $p(x)$ and the expected duration $m(x)$ for asymmetric Wiener processes in the interval $(-d, d)$, that is, for Wiener processes for which the infinitesimal means $\mu_{+}$and $\mu_{-}$, and infinitesimal variances $\sigma_{+}^{2}$ and $\sigma_{-}^{2}$ are not necessarily the same when $x>0$ or $x<0$. To confirm his results, he considered a random walk that converges to the Wiener process. Lefebvre's results were 
extended by Abundo [4] to general one-dimensional diffusion processes. However, Abundo did not obtain the quantities $p_{j}$ and $d_{j}$ for the corresponding discrete-time Markov chains. Also, it is worth mentioning that asymmetric diffusion processes need not be defined in an interval that includes the origin. A process defined in the interval $(a, b)$ can be asymmetric with respect to any $a<c<b$.

Next, Lefebvre and Guilbault [5] and Guilbault and Lefebvre [6] computed $p_{j}$ and $d_{j}$, respectively, for a discrete-time Markov chain that tends to the Ornstein-Uhlenbeck process. The authors also computed the quantity $p_{j}$ in the case when the Markov chain is asymmetric (as in Lefebvre [3]).

Asymmetric processes can be used in financial mathematics to model the price of a stock when, in particular, the infinitesimal variance (i.e., the volatility) tends to increase with the price of the stock. Indeed, it seems logical that the volatility is larger when the stock price $X(t)$ is very large than when it is close to zero. The prices of commodities, such as gold and oil, are also more volatile when they reach a certain level.

In order to check the validity of the expressions obtained by Abundo [4] for $p(x)$ and $m(x)$, it is important to obtain the corresponding quantities for the discrete-time Markov chains and then proceed by taking the limit as $\Delta x$ and $\Delta t$ decrease to zero appropriately. Moreover, the formulas that will be derived in the present paper are interesting in themselves, since in reality stock or commodity prices do not vary completely continuously.

First passage problems for Markov chains have many applications. For example, in neural networks, an important quantity is the interspike time, that is, the time between spikes of a firing neuron (which means that the neuron sends a signal to other neurons). Discretetime Markov chains have been used as models in this context, and the interspike time is the number of steps it takes the chain to reach the threshold at which firing occurs.

\section{Computation of the Probability $p_{j}$}

Assume first that $\Delta x=1$, so that the state space is $\{1,2, \ldots, N\}$ and the transition probabilities become

$$
p_{j, j+1}=\frac{1}{2 A}\left\{j^{2} \sigma^{2}+j \mu\right\}, \quad p_{j, j-1}=\frac{1}{2 A}\left\{j^{2} \sigma^{2}-j \mu\right\}, \quad p_{j, j}=1-\frac{j^{2} \sigma^{2}}{A}
$$

for $j \in\{2, \ldots, N-1\}$. The probability defined in (1.17) satisfies the following difference equation:

$$
p_{j}=p_{j, j+1} p_{j+1}+p_{j, j-1} p_{j-1}+p_{j, j} p_{j}
$$

That is,

$$
2 j p_{j}=(j+c) p_{j+1}+(j-c) p_{j-1}
$$

where $c=\mu / \sigma^{2}$. The boundary conditions are

$$
p_{1}=0, \quad p_{N}=1
$$


In the special case when $\mu=0,(2.3)$ reduces to the second-order difference equation with constant coefficients

$$
p_{j+1}=2 p_{j}-p_{j-1}
$$

We easily find that the (unique) solution that satisfies the boundary conditions (2.4) is

$$
p_{j}=\frac{j-1}{N-1} \quad \text { for } j=1,2, \ldots, N \text {. }
$$

Assume now that $\mu \neq 0$. Letting

$$
w_{j}:=p_{j+1}-p_{j}
$$

Equation (2.3) can be rewritten as

$$
(j+c) w_{j}=(j-c) w_{j-1}
$$

Using the mathematical software program Maple, we find that the solution of this first-order difference equation that satisfies the boundary condition $w_{1}=p_{2}$ is given by

$$
w_{j}=-\frac{p_{2}}{\pi} \sin [(2+c) \pi] c\left(c^{2}-1\right) \Gamma^{2}(c-1) \frac{\Gamma(j+1-c)}{\Gamma(j+1+c)},
$$

where $\Gamma$ is the gamma function.

Next, we must solve the first-order difference equation

$$
p_{j+1}-p_{j}=f(c) \frac{\Gamma(j+1-c)}{\Gamma(j+1+c)}
$$

where

$$
f(c):=-\frac{p_{2}}{\pi} \sin [(2+c) \pi] c\left(c^{2}-1\right) \Gamma^{2}(c-1),
$$

subject to the boundary conditions (2.4). We find that, if $c \neq 1 / 2$, then

$$
p_{j}=\frac{f(c)}{1-2 c}(j+c) \frac{\Gamma(j+1-c)}{\Gamma(j+1+c)}+f(c) \frac{c}{2 c-1} \frac{\Gamma(1-c)}{\Gamma(1+c)}+\kappa,
$$

where $\kappa$ is a constant. Applying the boundary conditions (2.4), we obtain that

$$
p_{j}=\frac{(j+c)(\Gamma(j+1-c) / \Gamma(j+1+c))-(1+c)(\Gamma(2-c) / \Gamma(2+c))}{(N+c)(\Gamma(N+1-c) / \Gamma(N+1+c))-(1+c)(\Gamma(2-c) / \Gamma(2+c))} \quad \text { for } j=1,2, \ldots, N
$$


Remark 2.1. When $c$ tends to $1 / 2$, the solution becomes

$$
p_{j}=\frac{\Psi(j+1 / 2)-2+\gamma+2 \ln 2}{\Psi(N+1 / 2)-2+\gamma+2 \ln 2},
$$

where $\gamma$ is Euler's constant and $\Psi$ is the digamma function defined by

$$
\Psi(z)=\frac{\Gamma^{\prime}(z)}{\Gamma(z)}
$$

Notice that

$$
\Psi(3 / 2)=2-\gamma-2 \ln 2,
$$

so that we indeed have $p_{1}=0$, and the solution (2.14) can be rewritten as

$$
p_{j}=\frac{\Psi(j+1 / 2)-\Psi(3 / 2)}{\Psi(N+1 / 2)-\Psi(3 / 2)} \quad \text { for } j=1,2, \ldots, N
$$

Now, in the general case when $\Delta x>0$, we must solve the difference equation

$$
\begin{aligned}
p_{j}= & \frac{1}{2 A}\left\{(1+j \Delta x)^{2} \sigma^{2}+(1+j \Delta x) \mu \Delta x\right\} p_{j+1} \\
& +\frac{1}{2 A}\left\{(1+j \Delta x)^{2} \sigma^{2}-(1+j \Delta x) \mu \Delta x\right\} p_{j-1} \\
& +\left(1-\frac{1}{A}(1+j \Delta x)^{2} \sigma^{2}\right) p_{j},
\end{aligned}
$$

which can be simplified to

$$
2(1+j \Delta x) p_{j}=[(1+j \Delta x)+c \Delta x] p_{j+1}+[(1+j \Delta x)-c \Delta x] p_{j-1} .
$$

The boundary conditions become

$$
p_{0}=0, \quad p_{k}=1
$$

When $\mu=0$ (which implies that $c=0$ ), the difference equation above reduces to the same one as when $\Delta x=1$, namely (2.5). The solution is

$$
p_{j}=\frac{j}{k} \quad \text { for } j=0,1, \ldots, k \text {. }
$$

Writing

$$
n=1+j \Delta x
$$


and using the fact that (by hypothesis) $N=1+k \Delta x$, we obtain that

$$
p_{n}=\frac{n-1}{N-1} \quad \text { for } n=1,1+\Delta x, \ldots, 1+k \Delta x=N
$$

Notice that this solution does not depend on the increment $\Delta x$. Hence, if we let $\Delta x$ decrease to zero and $k$ tend to infinity in such a way that $1+k \Delta x$ remains equal to $N$, we have that

$$
p_{n} \longrightarrow \frac{n-1}{N-1} \text { for } 1 \leq n \leq N
$$

which is the same as the function $p(x)$ in (1.6) when $c=0 / \sigma^{2}=0$.

Next, proceeding as above, we obtain that, if $c \neq 1 / 2$, the probability $p_{j}$ is given by

$$
p_{j}=\frac{(1+j \Delta x+c \Delta x)(\Gamma((1+(j+1) \Delta x-c \Delta x) / \Delta x) / \Gamma((1+(j+1) \Delta x+c \Delta x) / \Delta x))-\mathcal{A}}{(1+k \Delta x+c \Delta x)(\Gamma((1+(k+1) \Delta x-c \Delta x) / \Delta x) / \Gamma((1+(k+1) \Delta x+c \Delta x) / \Delta x))-\mathcal{A}},
$$

where $A$ denotes $(1+c \Delta x)(\Gamma((1+\Delta x-c \Delta x) / \Delta x) / \Gamma((1+\Delta x+c \Delta x) / \Delta x))$. In terms of $n$ and $N$, this expression becomes

$$
p_{n}=\frac{(n+c \Delta x)(\Gamma((n+\Delta x-c \Delta x) / \Delta x) /(\Gamma(n+\Delta x+c \Delta x) / \Delta x))-\mathcal{A}}{(N+c \Delta x)(\Gamma((N+\Delta x-c \Delta x) / \Delta x)) /(\Gamma((N+\Delta x+c \Delta x) / \Delta x))-\mathcal{A}}
$$

for $n \in\{1,1+\Delta x, \ldots, 1+k \Delta x=N\}$. The solution reduces to

$$
p_{n}=\frac{\Psi((2 n+\Delta x) / 2 \Delta x)-\Psi((2+\Delta x) / 2 \Delta x)}{\Psi((2 N+\Delta x) / 2 \Delta x)-\Psi((2+\Delta x) / 2 \Delta x)} \quad \text { if } c=1 / 2 .
$$

We can now state the following proposition.

Proposition 2.2. Let $n=1+j \Delta x$ for $j \in\{0,1, \ldots, k\}$, with $k$ such that $1+k \Delta x=N$. The probability $p_{n}$ that the discrete-time Markov chain defined in Section 1, starting from $n$, will hit $N$ before 1 is given by (2.23) if $\mu=0$, and by (2.26) if $c=\mu / \sigma^{2} \neq 0$. The value of $p_{n}$ tends to the function in (2.27) when $\mu / \sigma^{2}$ tends to $1 / 2$.

To complete this section, we will consider the case when $\Delta x$ decreases to zero. We have already mentioned that when $c=0$, the probability $p_{n}$ does not depend on $\Delta x$, and it corresponds to the function $p(x)$ in (1.6) with $c=0$.

Next, when $c=1 / 2$, making use of the formula

$$
\Psi(z) \sim \ln z \text { for } z \text { large, }
$$

we can write that

$$
\lim _{\Delta x \downarrow 0} p_{n}=\lim _{\Delta x \downarrow 0} \frac{\ln (2 n+\Delta x)-\ln (2+\Delta x)}{\ln (2 N+\Delta x)-\ln (2+\Delta x)}=\frac{\ln n}{\ln N} \quad \text { for } n \in[1, N] .
$$

Again, this expression corresponds to the function $p(x)$ given in (1.7), obtained when $c=1 / 2$. 
Finally, we have:

$$
\frac{\Gamma(z+a)}{\Gamma(z+b)} \alpha z^{a-b}\left(1+O\left(\frac{1}{z}\right)\right)
$$

as $|z|$ tends to infinity (if $|\operatorname{Arg}(z+a)|<\pi$ ). Hence, in the case when $c \neq 0,1 / 2$, we can write that

$$
\begin{aligned}
\lim _{\Delta x \downarrow 0} p_{n} & =\lim _{\Delta x \downarrow 0} \frac{(n+c \Delta x)(n+\Delta x)^{-2 c}-(1+c \Delta x)(1+\Delta x)^{-2 c}}{(N+c \Delta x)(N+\Delta x)^{-2 c}-(1+c \Delta x)(1+\Delta x)^{-2 c}} \\
& =\frac{n^{1-2 c}-1}{N^{1-2 c}-1}
\end{aligned}
$$

for $1 \leq n \leq N$. Therefore, we retrieve the formula for $p(x)$ in (1.6).

In the next section, we will derive the formulas that correspond to the function $m(x)$ in Section 1.

\section{Computation of the Mean Number of Transitions $d_{j}$ Needed to End the Game}

As in Section 2, we will first assume that $\Delta x=1$. Then, with $n=1+j$ for $j=0,1, \ldots, k$ (and $1+k=N$ ), the function $d_{n}$ satisfies the following second-order, linear, nonhomogeneous difference equation:

$$
d_{n}=p_{n, n+1} d_{n+1}+p_{n, n-1} d_{n-1}+p_{n, n} d_{n}+1 \quad \text { for } n=2, \ldots, N-1 \text {. }
$$

The boundary conditions are

$$
d_{1}=d_{N}=0 .
$$

We find that the difference equation can be rewritten as

$$
(n+c) d_{n+1}-2 n d_{n}+(n-c) d_{n-1}=-\frac{2 A}{n \sigma^{2}} .
$$

Let us now assume that $\mu=0$, so that we must solve the second-order, linear, nonhomogeneous difference equation with constant coefficients

$$
d_{n+1}-2 d_{n}+d_{n-1}=-\frac{2 A}{n^{2} \sigma^{2}} .
$$


With the help of the mathematical software program Maple, we find that the unique solution that satisfies the boundary conditions (3.2) is

$$
\begin{aligned}
d_{n}= & -\frac{n-1}{N-1} \frac{2 A}{\sigma^{2}}\left\{\Psi(N)+N \Psi(1, N)-(1-\gamma)-N\left(-1+\frac{\pi^{2}}{6}\right)\right\} \\
& +\frac{2 A}{\sigma^{2}}\left\{\Psi(n)+n \Psi(1, n)-(1-\gamma)-n\left(-1+\frac{\pi^{2}}{6}\right)\right\},
\end{aligned}
$$

where

$$
\Psi(1, x):=\frac{d}{d x} \Psi(x)
$$

is the first polygamma function.

Next, in the general case $\Delta x>0$, we must solve (with $c=0$ )

$$
d_{j+1}-2 d_{j}+d_{j-1}=-\frac{2 A}{(1+j \Delta x)^{2} \sigma^{2}}
$$

for $j=0,1, \ldots, k$. The solution that satisfies the boundary conditions

$$
d_{0}=d_{k}=0
$$

is given by

$$
\begin{aligned}
d_{j}= & -\frac{j}{k} \frac{2 A}{\sigma^{2}(\Delta x)^{3}}\left\{(1+k \Delta x) \Psi\left(1, \frac{1+k \Delta x}{\Delta x}\right)+\Delta x \Psi\left(\frac{1+k \Delta x}{\Delta x}\right)-\Psi\left(1, \frac{1}{\Delta x}\right)-\Delta x \Psi\left(\frac{1}{\Delta x}\right)\right\} \\
& +\frac{2 A}{\sigma^{2}(\Delta x)^{3}}\left\{(1+j \Delta x) \Psi\left(1, \frac{1+j \Delta x}{\Delta x}\right)+\Delta x \Psi\left(\frac{1+j \Delta x}{\Delta x}\right)-\Psi\left(1, \frac{1}{\Delta x}\right)-\Delta x \Psi\left(\frac{1}{\Delta x}\right)\right\} .
\end{aligned}
$$

In terms of $n:=1+j \Delta x$ and $N=1+k \Delta x$, this expression becomes

$$
\begin{aligned}
d_{n}= & -\frac{n-1}{N-1} \frac{2 A}{\sigma^{2}(\Delta x)^{3}}\left\{N \Psi\left(1, \frac{N}{\Delta x}\right)+\Delta x \Psi\left(\frac{N}{\Delta x}\right)-\Psi\left(1, \frac{1}{\Delta x}\right)-\Delta x \Psi\left(\frac{1}{\Delta x}\right)\right\} \\
& +\frac{2 A}{\sigma^{2}(\Delta x)^{3}}\left\{n \Psi\left(1, \frac{n}{\Delta x}\right)+\Delta x \Psi\left(\frac{n}{\Delta x}\right)-\Psi\left(1, \frac{1}{\Delta x}\right)-\Delta x \Psi\left(\frac{1}{\Delta x}\right)\right\}
\end{aligned}
$$

Finally, the mean duration of the game is obtained by multiplying $d_{n}$ by $\Delta t$. Making use of the fact that (see (1.14)) $\Delta t=(\Delta x)^{2} / A$, we obtain the following proposition. 
Proposition 3.1. When $\Delta x>0$ and $\mu=0$, the mean duration $D_{n}$ of the game is given by

$$
\begin{aligned}
D_{n}=- & \frac{n-1}{N-1} \frac{2}{\sigma^{2} \Delta x}\left\{N \Psi\left(1, \frac{N}{\Delta x}\right)+\Delta x \Psi\left(\frac{N}{\Delta x}\right)-\Psi\left(1, \frac{1}{\Delta x}\right)-\Delta x \Psi\left(\frac{1}{\Delta x}\right)\right\} \\
& +\frac{2}{\sigma^{2} \Delta x}\left\{n \Psi\left(1, \frac{n}{\Delta x}\right)+\Delta x \Psi\left(\frac{n}{\Delta x}\right)-\Psi\left(1, \frac{1}{\Delta x}\right)-\Delta x \Psi\left(\frac{1}{\Delta x}\right)\right\}
\end{aligned}
$$

for $n=1,1+\Delta x, \ldots, 1+k \Delta x=N$.

Next, using the fact that

$$
\Psi(x) \sim \ln x, \quad \Psi(1, x) \sim \frac{1}{x} \quad \text { for } x \text { large, }
$$

we obtain that, as $\Delta x$ decreases to zero and $1+k \Delta x$ remains equal to $N$,

$$
D_{n} \longrightarrow \frac{2}{\sigma^{2}}\left\{-\left(\frac{n-1}{N-1}\right) \ln N+\ln n\right\} \text { for } n \in[1, N]
$$

Notice that $D_{n}$ indeed corresponds to the function $m(x)$ given in (1.11) if $c=0$.

To complete our work, we need to find the value of the mean number of transitions $d_{j}$ in the case when $\mu \neq 0$ and $\Delta x>0$. To do so, we must solve the nonhomogeneous difference equation with nonconstant coefficients (3.3). We can obtain the general solution to the corresponding homogeneous equation. However, we then need to find a particular solution to the nonhomogeneous equation. This entails evaluating a difficult sum. Instead, we will use the fact that we know how to compute $d_{j}$ when $\mu=0$.

Let us go back to the geometric Brownian motion $\{X(t), t \geq 0\}$ defined in (1.1), and let us define, for $c \neq 1 / 2$,

$$
Y(t)=[X(t)]^{1-2 c}
$$

Then, we find (see Karlin and Taylor [7, page 173]) that $\{Y(t), t \geq 0\}$ remains a geometric Brownian motion, with infinitesimal variance $\sigma_{Y}^{2}=(1-2 c)^{2} \sigma^{2} y^{2}$, but with infinitesimal mean $\mu_{Y}=0$. In the case when $c=1 / 2$, we define

$$
Y(t)=\ln [X(t)],
$$

and we obtain that $\{Y(t), t \geq 0\}$ is a Wiener process with $\mu_{Y}=0$ and $\sigma_{Y}^{2}=\sigma^{2}$.

Remark 3.2. When $c=1 / 2$, we find that $\{X(t), t \geq 0\}$ can be expressed as the exponential of a Wiener process $\{W(t), t \geq 0\}$ having infinitesimal mean $\mu_{W}=0$ and infinitesimal variance $\sigma_{W}^{2}=\sigma^{2}$. 
When we make the transformation $Y(t)=[X(t)]^{1-2 c}$, the interval $[1, N]$ becomes $\left[1, N^{1-2 c}\right]$, respectively $\left[N^{1-2 c}, 1\right]$, if $c<1 / 2$, respectively $c>1 / 2$. Assume first that $c<1 / 2$. We have (see (1.2))

$$
\tau(x)=\inf \left\{t>0: Y(t)=1 \text { or } N^{1-2 c} \mid Y(0)=x^{1-2 c}\right\}
$$

Now, we consider the discrete-time Markov chain with state space $\{1,1+\Delta x, \ldots, 1+$ $\left.k \Delta x=N^{1-2 c}\right\}$ and transition probabilities given by (1.13). Proceeding as above, we obtain the expression in (3.9) for the mean number of transitions $d_{j}$ from state $1+j \Delta x$. This time, we replace $1+j \Delta x$ by $n^{1-2 c}$ and $1+k \Delta x$ by $N^{1-2 c}$, so that

$$
\begin{aligned}
d_{n}= & -\frac{n^{1-2 c}-1}{N^{1-2 c}-1} \frac{2 A}{\sigma^{2}(\Delta x)^{3}}\left\{N^{1-2 c} \Psi\left(1, \frac{N^{1-2 c}}{\Delta x}\right)+\Delta x \Psi\left(\frac{N^{1-2 c}}{\Delta x}\right)-\Psi\left(1, \frac{1}{\Delta x}\right)-\Delta x \Psi\left(\frac{1}{\Delta x}\right)\right\} \\
& +\frac{2 A}{\sigma^{2}(\Delta x)^{3}}\left\{n^{1-2 c} \Psi\left(1, \frac{n^{1-2 c}}{\Delta x}\right)+\Delta x \Psi\left(\frac{n^{1-2 c}}{\Delta x}\right)-\Psi\left(1, \frac{1}{\Delta x}\right)-\Delta x \Psi\left(\frac{1}{\Delta x}\right)\right\}
\end{aligned}
$$

for $n=1,(1+\Delta x)^{1 /(1-2 c)}, \ldots,(1+k \Delta x)^{1 /(1-2 c)}=N$.

Assume that each displacement takes

$$
\Delta t=\frac{(\Delta x)^{2}}{(1-2 c)^{2} A}
$$

time units. Taking the limit as $\Delta x$ decreases to zero (and $k \rightarrow \infty$ ), we obtain (making use of the formulas in (3.12)) that

$$
D_{n} \longrightarrow \frac{2}{(1-2 c) \sigma^{2}}\left\{-\left(\frac{n^{1-2 c}-1}{N^{1-2 c}-1}\right) \ln N+\ln n\right\} \text { for } n \in[1, N]
$$

This formula corresponds to the function $m(x)$ in (1.11) when $c<1 / 2$.

When $c>1 / 2$, we consider the Markov chain having state space

$$
\left\{\frac{1}{N^{2 c-1}}=\frac{1}{1+k \Delta x}, \frac{1}{1+(k-1) \Delta x}, \ldots, \frac{1}{1+\Delta x}, 1\right\}
$$

(and transition probabilities given by (1.13)). To obtain $d_{j}$, we must again solve the difference equation (3.7), subject to the boundary conditions $d_{0}=d_{k}=0$. However, once we have obtained the solution, we must now replace $1+j \Delta x$ by $(1+j \Delta x)^{-1}$ (and $1+k \Delta x$ by $\left.(1+k \Delta x)^{-1}\right)$. Moreover, because

$$
j=\frac{(1+j \Delta x)-1}{\Delta x}
$$


we replace $j$ by

$$
\frac{1 /(1+j \Delta x)-1}{\Delta x}=-\frac{j}{1+j \Delta x}
$$

(and similarly for $k$ ).

Remark 3.3. The quantity $d_{j}$ here actually represents the mean number of steps needed to end the game when the Markov chain starts from state $1 /(1+j \Delta x)$, with $j \in\{0, \ldots, k\}$.

We obtain that

$$
\begin{gathered}
d_{j}=-\frac{j}{1+j \Delta x} \frac{1+k \Delta x}{k} \frac{2 A}{\sigma^{2}(\Delta x)^{3}}\left\{\frac{1}{1+k \Delta x} \Psi\left(1, \frac{1}{(1+k \Delta x) \Delta x}\right)+\Delta x \Psi\left(\frac{1}{(1+k \Delta x) \Delta x}\right)\right. \\
\left.+\Psi\left(1, \frac{1}{\Delta x}\right)-\Delta x \Psi\left(\frac{1}{\Delta x}\right)\right\} \\
+\frac{2 A}{\sigma^{2}(\Delta x)^{3}}\left\{\frac{1}{1+j \Delta x} \Psi\left(1, \frac{1}{(1+j \Delta x) \Delta x}\right)+\Delta x \Psi\left(\frac{1}{(1+j \Delta x) \Delta x}\right)\right. \\
\left.-\Psi\left(1, \frac{1}{\Delta x}\right)-\Delta x \Psi\left(\frac{1}{\Delta x}\right)\right\} .
\end{gathered}
$$

Next, since $N^{2 c-1}=1+k \Delta x$, setting $n^{2 c-1}=1+j \Delta x$ we deduce from the previous expression that

$$
\begin{aligned}
d_{n}= & -\frac{n^{2 c-1}-1}{n^{2 c-1}} \frac{N^{2 c-1}}{N^{2 c-1}-1} \frac{2 A}{\sigma^{2}(\Delta x)^{3}}\left\{\frac{1}{N^{2 c-1}} \Psi\left(1, \frac{N^{1-2 c}}{\Delta x}\right)\right. \\
& \left.+\Delta x \Psi\left(\frac{N^{2 c-1}}{\Delta x}\right)-\Psi\left(1, \frac{1}{\Delta x}\right)-\Delta x \Psi\left(\frac{1}{\Delta x}\right)\right\} \\
+ & \frac{2 A}{\sigma^{2}(\Delta x)^{3}}\left\{\frac{1}{n^{2 c-1}} \Psi\left(1, \frac{n^{1-2 c}}{\Delta x}\right)+\Delta x \Psi\left(\frac{n^{2 c-1}}{\Delta x}\right)-\Psi\left(1, \frac{1}{\Delta x}\right)-\Delta x \Psi\left(\frac{1}{\Delta x}\right)\right\}
\end{aligned}
$$

for $n \in\left\{1,(1+\Delta x)^{1 /(2 c-1)}, \ldots,(1+k \Delta x)^{1 /(2 c-1)}=N\right\}$.

Finally, if we assume, as above, that each step of the Markov chain takes

$$
\Delta t=\frac{(\Delta x)^{2}}{(2 c-1)^{2} A}
$$

time units, we find that, when $\Delta x$ decreases to zero, the mean duration of the game tends to

$$
D_{n}=\frac{2}{(2 c-1) \sigma^{2}}\left\{\ln n-\left(\frac{1-n^{1-2 c}}{1-N^{1-2 c}}\right) \ln N\right\} \quad \text { for } n \in[1, N]
$$

This last expression is equivalent to the formula for $m(x)$ in $(1.11)$ when $c>1 / 2$. 
Remark 3.4. Actually, the formula for $m(x)$ is the same whether $c<1 / 2$ or $c>1 / 2$.

At last, in the case when $c=1 / 2$, we consider the random walk with state space $\{0, \Delta x, \ldots, k \Delta x=\ln N\}$ and transition probabilities

$$
\begin{gathered}
p_{j \Delta x,(j+1) \Delta x}=p_{j \Delta x,(j-1) \Delta x}=\frac{\sigma^{2}}{2 A} \\
p_{j \Delta x, j \Delta x}=1-\frac{\sigma^{2}}{A}
\end{gathered}
$$

Then, we must solve the nonhomogeneous difference equation

$$
d_{j+1}-2 d_{j}+d_{j-1}=-\frac{2 A}{\sigma^{2}},
$$

subject to the boundary conditions $d_{0}=d_{k}=0$. We find that

$$
d_{j}=-\frac{j}{k} \frac{A}{\sigma^{2}} k(1-k)+\frac{A}{\sigma^{2}} j(1-j) .
$$

With $\ln n:=j \Delta x$ and $\ln N=k \Delta x$, we get that

$$
d_{n}=\frac{A}{\sigma^{2}(\Delta x)^{2}}\{\ln n(\ln N-\ln n)\}
$$

for $n \in\left\{1, e^{\Delta x}, \ldots, e^{k \Delta x}=N\right\}$. Assuming that $\Delta t=(\Delta x)^{2} / A$, we deduce at once that, as $\Delta x$ decreases to zero,

$$
D_{n} \longrightarrow \frac{1}{\sigma^{2}}\{\ln n(\ln N-\ln n)\} \quad \text { for } n \in[1, N]
$$

Thus, we retrieve the formula (1.12) for $m(x)$ when $c=1 / 2$.

We can now state the following proposition.

Proposition 3.5. If the state space of the Markov chain is

$$
\left\{1,(1+\Delta x)^{1 /(1-2 c)}, \ldots,(1+k \Delta x)^{1 /(1-2 c)}=N\right\},
$$

respectively,

$$
\left\{1,(1+\Delta x)^{1 /(2 c-1)}, \ldots,(1+k \Delta x)^{1 /(2 c-1)}=N\right\}
$$

where $c<1 / 2$, respectively $c>1 / 2$, and the transition probabilities are those in (1.13), then the value of the mean number of steps $d_{n}$ needed to end the game is given by (3.17), respectively, (3.24). If $n \in\left\{1, e^{\Delta x}, \ldots, e^{k \Delta x}=N\right\}$ and the transition probabilities are the ones in (3.27), then the value of $d_{n}$ is given by (3.30). 


\section{Concluding Remarks}

We have obtained explicit and exact formulas for the quantities $p_{j}$ and $d_{j}$ defined respectively in (1.17) and (1.18) for various discrete-time Markov chains that converge, at least in a finite interval, to a geometric Brownian motion. In the case of the probability $p_{j}$ of hitting the boundary $N$ before 1 , because the appropriate difference equation is homogeneous, we were able to compute this probability for any value of $c=\mu / \sigma^{2}$ by considering a Markov chain with state space $\{1,(1+\Delta x), \ldots,(1+k \Delta x)=N\}$. However, to obtain $d_{j}$ we first solved the appropriate difference equation when $c=0$. Then, making use of the formula that we obtained, we were able to deduce the solution for any $c \in \mathbb{R}$ by considering a Markov chain that converges to a transformation of the geometric Brownian motion. The transformed process was a geometric Brownian motion with $\mu=0$ (if $c \neq 1 / 2)$, or a Wiener process with $\mu=0$ (if $c=1 / 2$ ). In each case, we showed that the expression that we derived tends to the corresponding quantity for the geometric Brownian motion. In the case of the mean duration of the game, the time increment $\Delta t$ had to be chosen suitably.

As is well known, the geometric Brownian motion is a very important model in financial mathematics, in particular. In practice, stock or commodity prices vary discretely over time. Therefore, it is interesting to derive formulas for $p_{j}$ and $d_{j}$ for Markov chains that are as close as we want to the diffusion process.

Now that we have computed explicitly the value of $p_{j}$ and $d_{j}$ for Markov chains having transition probabilities that involve parameters $\mu$ and $\sigma^{2}$ that are the same for all the states, we could consider asymmetric Markov chains. For example, at first the state space could be $\left\{1, \ldots, N_{1}, \ldots, N_{2}\right\}$, and we could have

$$
\mu= \begin{cases}\mu_{1} & \text { if } n \in\left\{1, \ldots, N_{1}-1\right\}, \\ \mu_{2} & \text { if } n \in\left\{N_{1}+1, \ldots, N_{2}\right\}\end{cases}
$$

(and similarly for $\sigma^{2}$ ). When the Markov chain hits $N_{1}$, it goes to $N_{1}+1$, respectively $N_{1}-1$, with probability $p_{0}$, respectively $1-p_{0}$. By increasing the state space to $\left\{1,1+\Delta x, \ldots, 1+k_{1} \Delta x=\right.$ $\left.N_{1}, \ldots, 1+k_{2} \Delta x=N_{2}\right\}$, and taking the limit as $\Delta x$ decreases to zero (with $k_{1}$ and $k_{2}$ going to infinity appropriately), we would obtain the quantities that correspond to $p_{j}$ and $d_{j}$ for an asymmetric geometric Brownian motion. The possibly different values of $\sigma^{2}$ depending on the state $n$ of the Markov chain reflect the fact that volatility is likely to depend on the price of the stock or the commodity.

Finally, we could try to derive the formulas for $p_{j}$ and $d_{j}$ for other discrete-time Markov chains that converge to important one-dimensional diffusion processes.

\section{References}

[1] M. Lefebvre, Applied Stochastic Processes, Springer, New York, NY, USA, 2007.

[2] D. R. Cox and H. D. Miller, The Theory of Stochastic Processes, Methuen, London, UK, 1965.

[3] M. Lefebvre, "First passage problems for asymmetric Wiener processes," Journal of Applied Probability, vol. 43, no. 1, pp. 175-184, 2006.

[4] M. Abundo, "First-passage problems for asymmetric diffusions and skew-diffusion processes," Open Systems and Information Dynamics, vol. 16, no. 4, pp. 325-350, 2009.

[5] M. Lefebvre and J.-L. Guilbault, "First hitting place probabilities for a discrete version of the OrnsteinUhlenbeck process," International Journal of Mathematics and Mathematical Sciences, vol. 2009, Article ID 909835, 12 pages, 2009. 
[6] J.-L. Guilbault and M. Lefebvre, "On a non-homogeneous difference equation from probability theory," Tatra Mountains Mathematical Publications, vol. 43, pp. 81-90, 2009.

[7] S. Karlin and H. M. Taylor, A Second Course in Stochastic Processes, Academic Press, New York, NY, USA, 1981. 


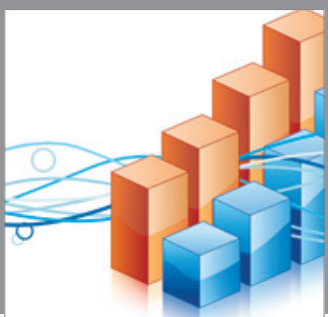

Advances in

Operations Research

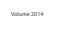

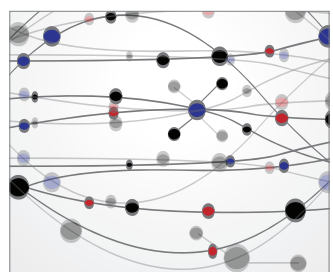

\section{The Scientific} World Journal
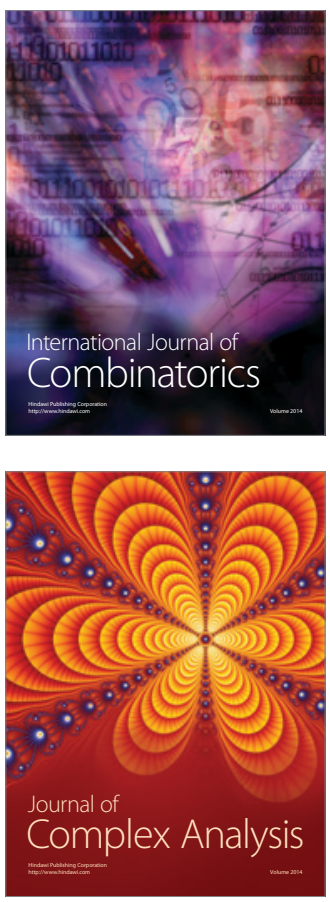

International Journal of

Mathematics and

Mathematical

Sciences
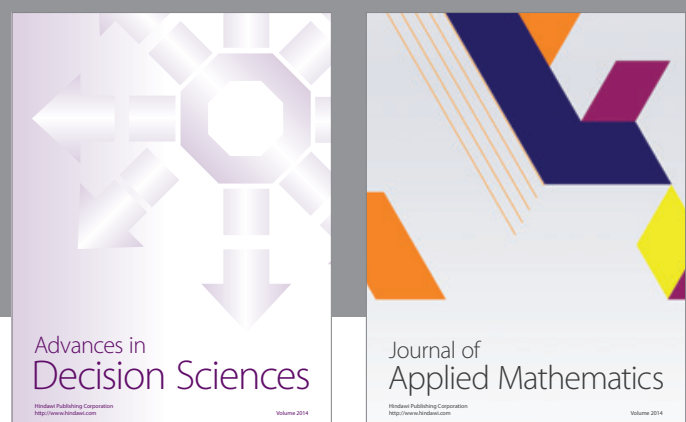

Journal of

Applied Mathematics
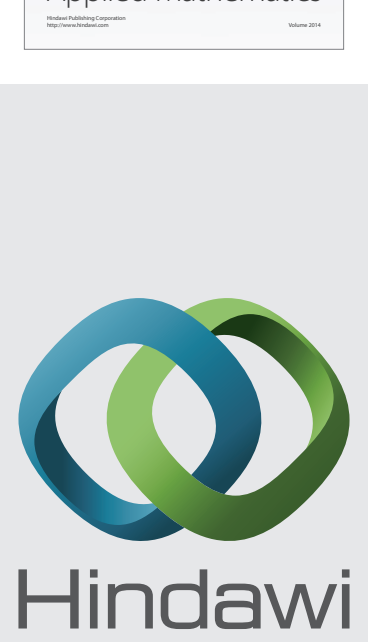

Submit your manuscripts at http://www.hindawi.com
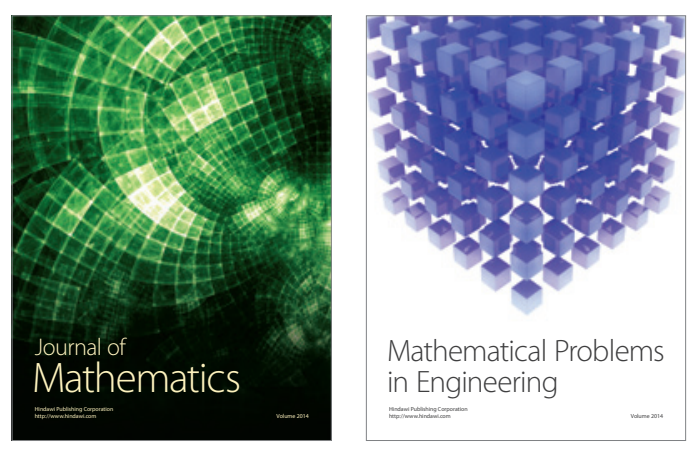

Mathematical Problems in Engineering
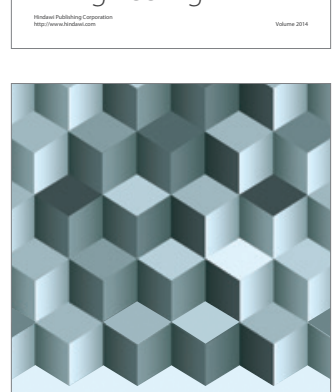

Journal of

Function Spaces
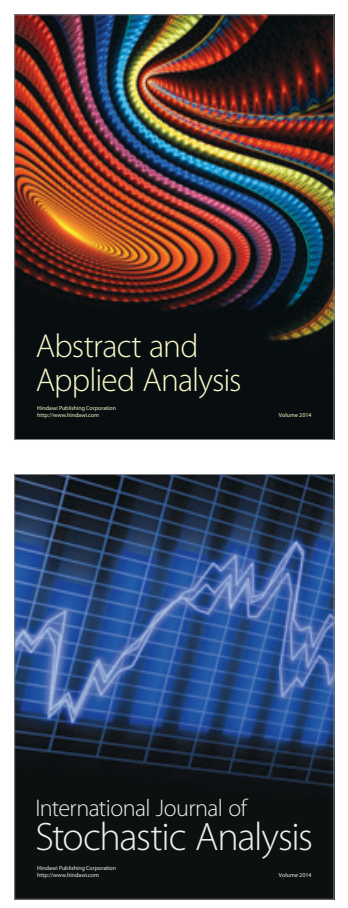

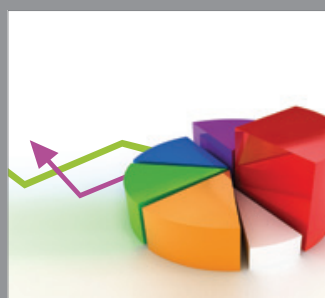

ournal of

Probability and Statistics

Promensencen
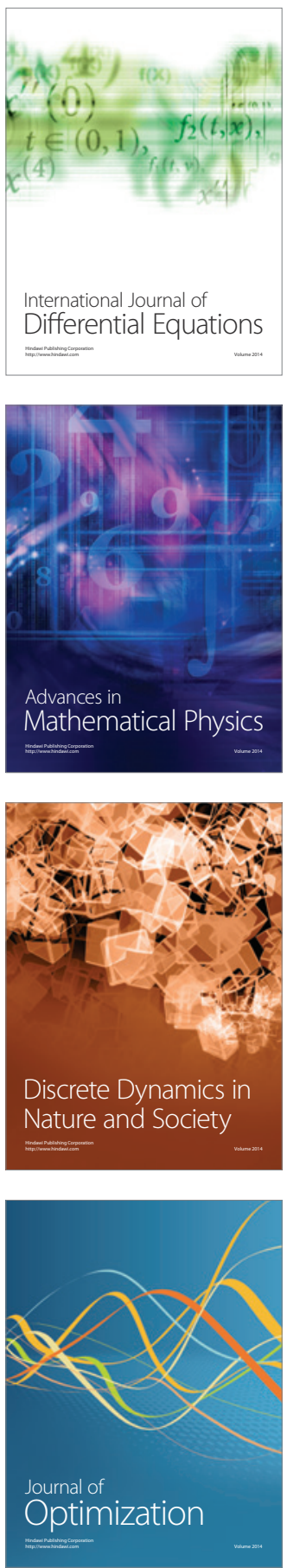